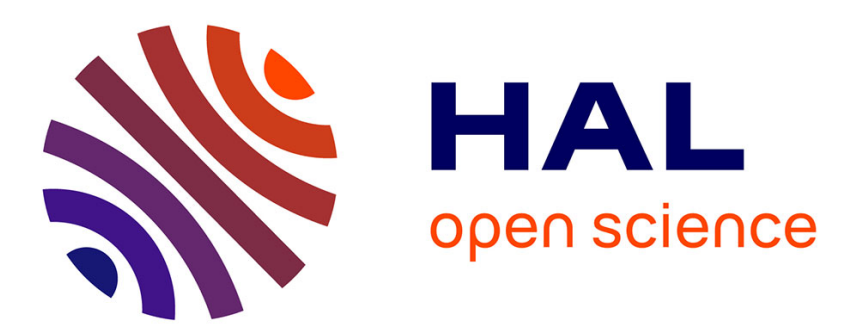

\title{
Qualitative Analysis of Mammalian Circadian Oscillations: Cycle Dynamics and Robustness
}

Ousmane Diop, Madalena Chaves, Laurent Tournier

\section{To cite this version:}

Ousmane Diop, Madalena Chaves, Laurent Tournier. Qualitative Analysis of Mammalian Circadian Oscillations: Cycle Dynamics and Robustness. CMSB 2020 - 18th International Conference on Computational Methods in Systems Biology, Sep 2020, Konstanz, Germany. pp.176-192, 10.1007/978-3030-60327-4_10. hal-03105583

\section{HAL Id: hal-03105583 \\ https://hal.inria.fr/hal-03105583}

Submitted on 11 Jan 2021

HAL is a multi-disciplinary open access archive for the deposit and dissemination of scientific research documents, whether they are published or not. The documents may come from teaching and research institutions in France or abroad, or from public or private research centers.
L'archive ouverte pluridisciplinaire HAL, est destinée au dépôt et à la diffusion de documents scientifiques de niveau recherche, publiés ou non, émanant des établissements d'enseignement et de recherche français ou étrangers, des laboratoires publics ou privés. 


\title{
Qualitative analysis of mammalian circadian oscillations: cycle dynamics and robustness ${ }^{\star}$
}

\author{
Ousmane Diop ${ }^{1}$, Madalena Chaves $^{2}$, and Laurent Tournier ${ }^{1}$ \\ 1 MaIAGE, INRAE, Université Paris-Saclay, 78350 Jouy-en-Josas, France. \\ ousmane.diop@inrae.fr \\ 2 Université Côte d'Azur, Inria, INRAE, CNRS, Sorbonne Université, \\ Biocore team, Sophia Antipolis, France
}

\begin{abstract}
In asynchronous Boolean models, periodic solutions are represented by terminal strongly connected graphs, which are typically composed of hundreds of states and transitions. For biological systems, it becomes a challenging task to compare such mathematical objects with biological knowledge, or interpret the transitions inside an attractor in terms of the sequence of events in a biological cycle. A recent methodology generates summary graphs to help visualizing complex asynchronous attractors and order the dynamic progression based on known biological data. In this article we apply this method to a Boolean model of the mammalian circadian clock, for which the summary graph recovers the main phases of the cycle, in the expected order. It also provides a detailed view of the attractor, suggesting improvements in the design of the model's logical rules and highlighting groups of transitions that are essential for the attractor's robustness.
\end{abstract}

Keywords: Mammalian circadian clock · Asynchronous Boolean network · Complex attractor · Summary graph

\section{Introduction}

The analysis of periodic orbits and their properties remains a most challenging problem in dynamical systems theory. Many living systems exhibit periodical dynamics and the current literature covers a large diversity of mathematical models used to represent, explore, and study the mechanisms leading to physical or biological rhythms [7]. A thorough analysis of such cyclic attractors opens the door to a whole family of meaningful questions related to the robustness of the oscillatory behavior, the estimation and control of the period or amplitude of oscillations in terms of the parameters of the system, the location of the orbit in the state space, etc.

Very little is known on how to express the properties of a periodic solution in terms of the system's parameters but qualitative models, such as piecewise linear or Boolean models, suggest some ideas. Piecewise linear systems partition the

\footnotetext{
* Supported by the ANR (French agency for research) through project ICycle ANR16-CE33-0016-01.
} 
state space into regions where solutions of the system can be explicitly computed leading, in some examples, to the estimation of the period and other quantities in terms of the parameters $[13,14]$. Boolean models provide an ideal framework to analyze qualitative dynamical properties by enabling algorithmic approaches to characterize, for instance, the location of a periodic orbit in the state space, or the influence of the interaction graph on the dynamical behavior $[21,17]$.

In an asynchronous Boolean network, a periodic orbit corresponds to a terminal strongly connected component of the state transition graph. If the computation of such an object is not an issue form a theoretical point of view, its size can grow very large, strongly limiting the biological interpretation of the states and transitions inside the attractor. To tackle this issue, general approaches can be used such as model checking techniques [23]. Recently, a dedicated method developed by Diop et al. [5] proposes to generate a summary graph of an asynchronous attractor, based on a classification of its states according to experimentally observed phases of the biological system. The summary graph provides a qualitative view of the general progression along the periodic orbit, capturing the underlying dynamics within the cyclic attractor. In [5] it was successfully applied to a Boolean model of the mammalian cell cycle [6].

In this paper, we propose to apply the summary graph method to the mammalian circadian clock, a biological rhythm which is based on the interactions among five main proteins CLOCK:BMAL1, REV-ERB $\alpha$, ROR, PER, and CRY. The core of the clock mechanism is formed by three feedback loops. First, the BMAL1 complex promotes the transcription of Per and Cry genes. The corresponding proteins bind to form a complex PER:CRY, which then translocates to the nucleus where it will block the transcriptional activity of BMAL1, thus forming a first negative feedback loop. In addition, BMAL1 also promotes the transcription of the two genes Rev-erb and Ror. Eventually, Bmal1 transcription will be inhibited by REV-ERB and activated by ROR, leading to a second negative loop and a new positive loop, respectively. The periodic behavior of the clock system is determined in large part by the phase opposition between CLOCK:BMAL1 and PER:CRY, which corresponds to the day/night succession (BMAL1 peaks during the day).

Examples of Boolean models for circadian clocks include one for the plant Arabidopsis thaliana [1] and a compact mammalian clock model [4] which reproduces the interplay between the negative feedback loops induced by BMAL1 activity. However, our present objective is to have a deeper understanding of the circadian cycle recently developed by Almeida et al. [2]. This is a continuous model of the core clock mechanism that faithfully reproduces the circadian rhythm, by including not only the five main proteins but also their transcription regulated by clock controlled elements (CCE). To use the graph method [5], we first construct a Boolean version of the continuous model in [2] that exhibits one cyclic attractor and, in addition, correctly reproduces the effect of some well known gene knock-outs. Next, Section 3 analyzes the cyclic attractor with the summary graph method, by classifying groups of Boolean states according to their corresponding circadian time zones. Finally, in Section 4 the summary 
graph is further used to identify key groups of transitions within the attractor and relate them back to parts of the network's logical rules. This analysis has two outcomes, first suggesting an improved Boolean rule for one of the variables where the model lacked clear information. As a second outcome, our analysis predicts that some transitions, while allowing some short-circuits between phases, seem to contribute to the overall robustness of the attractor and globally ensure the good progression of the cell clock.

\section{Proposing a new Boolean model of the circadian clock}

As evoked previously, the following Boolean model of the mammalian circadian clock is highly inspired by the continuous model developed in [2]. In addition to the three feedback loops already described, this model takes into account the transcription of the five clock proteins, each regulated through a particular combination of transcription factors. The latter bind to specific sites on the promoters, called clock controlled elements (CCEs): Ebox (enhancer box), Dbox (DBP/E4BP4 response element), and Rbox (REV-ERB/ROR response element). With the introduction of Dbox (which activates both REV-ERB and PER), two new proteins are added to the model, DBP and E4BP4, each also regulated by one of the CCE.

A complete and detailed justification of the continuous model assumptions and construction can be found in [2], but we provide a brief summary of the eight variables and corresponding differential equations in Table 1.

\subsection{Construction of the Boolean model}

The design of a Boolean or of a continuous model for the same biological system differs in some fundamental aspects and, in general, there is no direct equivalence between terms in the two frameworks. For instance, activation and inhibition links typically have clear logical representations, but the effects of detailed mass-action kinetics or mass conservation laws are harder to represent in a Boolean model, and may require the definition of new variables. The purpose of our Boolean model is to transcribe as closely as possible the interactions in the continuous model in [2], as described below. The continuous equations and corresponding logical rules are shown in Table 1, for a clear comparison between the two models.

In [2], there are three CCE named Dbox, Ebox, and Rbox, each responding to the conjugation of two components, an activator and an inhibitor. More precisely, Dbox is activated by DBP and inhibited by E4, Ebox is activated by Bmal and inhibited by CRY and Rbox is activated by ROR and inhibited by REV. The continuous equations are formed by a synthesis term depending on one or two $\mathrm{CCE}$ and a degradation term. The $C R Y, P E R$ and $P C$ equations also contain the binding and dissociation terms denoted $M_{P C}$ in Table 1 .

In general, Boolean variables are assumed to degrade when not updated in the next step and, therefore, linear degradation terms do not appear explicitly. Thus, 
Table 1. Differential equations of the circadian clock model in [2] and corresponding logical rules in the Boolean model. The term $M_{P C}=\gamma_{p c} P E R \cdot C R Y-\gamma_{c p} P C$ represents the kinetics of the complex binding: PER+CRY $\rightleftharpoons \mathrm{PC}$ (mass action law). We use classical Boolean operators: $X \vee Y(X$ or $Y), X \wedge Y(X$ and $Y)$ and $\bar{X}(\operatorname{not} X)$.

\begin{tabular}{ll}
\hline Continuous equation & Logical rule \\
\hline$d B M A L 1 / d t=R b o x-\gamma_{b p} B M A L 1 \cdot P C$ & $B m a l^{\prime}=R b o x \wedge \overline{P C}$ \\
$d R O R / d t=E b o x+R b o x-\gamma_{r o r} R O R$ & $R O R^{\prime}=R b o x_{2}$ \\
& $R O R 2^{\prime}=E b o x \wedge R b o x \wedge R O R$ \\
$d R E V / d t=2 E b o x+D b o x-\gamma_{r e v} R E V$ & $R E V^{\prime}=E b o x \vee R E V 2$ \\
& $R E V 2^{\prime}=E b o x \wedge D b o x \wedge R E V$ \\
$d D B P / d t=E b o x-\gamma_{d b p} D B P$ & $D B P^{\prime}=E b o x$ \\
$d E 4 / d t=2 R b o x-\gamma_{e 4} E 4$ & $E 4^{\prime}=R b o x$ \\
$d C R Y / d t=E b o x+2 R b o x-M_{P C}-\gamma_{c} C R Y$ & $C R Y^{\prime}=E b o x \vee C R Y 2$ \\
& $C R Y 2^{\prime}=E b o x \wedge R b o x \wedge C R Y$ \\
$d P E R / d t=E b o x+D b o x-M_{P C}-\gamma_{p} P E R$ & $P E R^{\prime}=E b o x \vee D b o x$ \\
$d P C / d t=M_{P C}-\gamma_{b p} B M A L 1 \cdot P C$ & $P C^{\prime}=P E R \wedge C R Y$
\end{tabular}

a naive approach to construct a corresponding Boolean model is to combine the synthesis terms as logical conjugations or disjunctions of the given variables and directly obtain the rule, for instance: $D B P^{\prime}=E b o x$ or $E 4^{\prime}=R b o x$. However, not all continuous equations follow this simple construction and other properties that strongly contribute to the dynamics must be taken into account.

Indeed, (i) some variables have nonlinear degradation (cf. $B M A L 1$ ), (ii) others contain mass-action terms (cf. $C R Y, P E R, P C$ ) and (iii) three of the variables are regulated by two CCEs and are themselves regulators of CCEs $(R O R$, $R E V, C R Y)$. This last property implies the existence of different thresholds for the different regulatory activities. For this reason, ROR, $R E V$, and $C R Y$ are assumed to have an extra discrete level, here represented by extra Boolean variables $R O R 2, R E V 2$, and $C R Y 2$ (following [24]), under the assumption

$$
x=\left\{\begin{array}{l}
0 \text { when } X=X 2=0, \\
1 \text { when } X=1, X 2=0, \\
2 \text { when } X=X 2=1
\end{array}\right.
$$

The states corresponding to $X=0, X 2=1$ have no biological meaning (also called "forbidden") and trajectories towards these states from the other "biological" states must be excluded. This can be achieved by using the method described in [3] which complements the rules for $X^{\prime}=(\cdots)$ and $X 2^{\prime}=(\cdots)$ as follows: $X^{\prime}=(\cdots) \vee X 2$ and $X 2^{\prime}=(\cdots) \wedge X$. Since the CCEs appear in additive 
form in the continuous equations, we assume that only one CCE is sufficient to trigger the activity of $X$, while the two CCEs are needed to trigger the activity of $X 2$. The order in which the CCEs are activated and in turn activate each variable, was decided by comparing to the continuous solutions. The expressions for the CCEs are written as logical conjunctions:

$$
\begin{aligned}
& E b o x=B m a l \wedge \overline{C R Y 2}, \quad \text { Dbox }=D B P \wedge \overline{E 4}, \\
& R b o x=R O R \wedge \overline{R E V,} \quad R_{b o x_{2}}=R O R 2 \wedge \overline{R E V} .
\end{aligned}
$$

In the continuous model, there is only one nonlinear degradation term, corresponding to the inhibition of BMAL1 transcription by PC $\left(-\gamma_{b p} B M A L 1 \cdot P C\right)$. Accordingly, we assume $\mathrm{Bmal}$ is explicitly repressed by $P C$ but, conversely, PC is not strongly affected (indeed, our analysis showed that a rule of the form $P C=P E R \wedge C R Y \wedge \overline{B m a l}$ prevents oscillatory behavior).

The binding of PER and $C R Y$ to form the complex $P C$ is described by mass-action kinetics in the continuous model (see term $M_{P C}$ in Table 1). In the Boolean model, for simplicity, we assumed that $P C$ is produced when both $P E R$ and $C R Y$ are available, leading to the rule $P C=P E R \wedge C R Y$, but no explicit effect from $P C$ on $P E R$ or $C R Y$. Although there is no systematic way to translate mass-action kinetics or other mass conservation laws into Boolean factors, and these are usually treated on a case-by-case basis, we will see in Part 4 that the analysis by the summary graph method suggests a refinement of the $P E R$ rule. This refinement can be interpreted as a more suitable way to include the mass-action terms into the circadian clock Boolean model.

In this way, we obtained a qualitative multi-valued model which closely translates the differential model of [2]. The multi-valued model is equivalent to the 11-dimensional Boolean network depicted in Table 2, to which the methodology in [5] can now be applied.

Table 2. Logical rules of the Boolean clock model, with 11 variables.

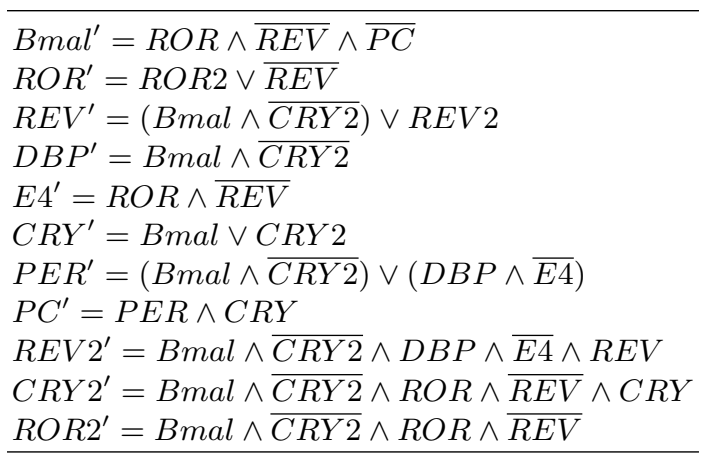




\subsection{First dynamical analysis of the Boolean model}

To compute and analyze the dynamical behavior of a Boolean model $X_{i}^{\prime}=$ $F_{i}\left(X_{1}, \ldots, X_{n}\right), i=1, \ldots, n$, we need to specify an updating order for the variables. Applying the logical rules in the order defined by the updating schedule leads to a state transition graph (STG) with $2^{n}$ states, where a sequence of transitions represents a trajectory of the system. To analyze the STG, we first compute its strongly connected components (SCCs) which are defined as sets of states $C$, such that for every pair of states $x, y \in C$, there exist two paths (or sequences of transitions) in $C$ leading from $x$ to $y$ and from $y$ to $x$. SCCs may consist of single or multiple states and may have incoming and outgoing transitions, but two distinct SCCs can not be mutually connected, otherwise they would form a single SCC. The asymptotic behavior of the system is thus characterized by the SCCs without outgoing transitions, also called terminal SCCs or attractors. An attractor with multiple states represents a periodic orbit of the system.

As a preliminary analysis, we considered the basic synchronous updating schedule, where all variables are simultaneously updated: $X_{i}[t+1]=F_{i}(X[t])$. The synchronous STG of the model contains a single attractor, composed of only five states. This simple cyclic attractor captures the Bmal/PER:CRY phase opposition, a central feature in the circadian clock (see Figure 1).

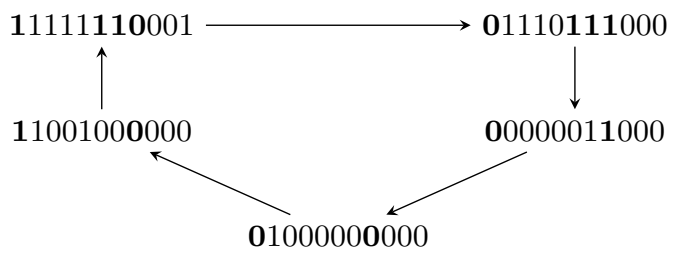

Fig. 1. Synchronous attractor of the Boolean model (variables are ordered as in Table 2). Bold digits indicate the succession of states corresponding to the $\mathrm{Bmal} / \mathrm{PC}$ phase opposition: in the left column, Bmal is expressed while $P C$ is turned off, the former leading to expression of $P E R$ and $C R Y$ (top left); next $P C$ becomes expressed and Bmal is turned off (right column), and $C R Y$ and PER eventually turn off; at the bottom both $P C$ and $B m a l$ are off, before a new cycle begins.

In this paper, we will prefer an asynchronous updating schedule as it is much more realistic from a biological perspective. In this scheme, at most one variable is updated at each instant. To construct the STG for this asynchronous schedule, for each state $X=\left(X_{1}, \ldots, X_{n}\right)$ define the subset of variables $I_{X}=\left\{i: X_{i} \neq\right.$ $\left.F_{i}(X)\right\}$. Then, for each $i \in I_{X}$, add a transition $X \rightarrow Y$ where $Y_{i}=F_{i}(X)$ and $Y_{j}=X_{j}$ for all $j \neq i$. More details on the asynchronous strategy can be found for instance in [21]. From a biological point of view, asynchronous updating is preferable as it allows for variability and different timescales in the network interactions. From a graph theoretical point of view, synchronous and asynchronous 
STGs are very different as the asynchronous STG is non deterministic, i.e. a state no longer has one and only one successor. Usually, this generates lots of transitions, making biological interpretation more difficult.

When applied to our circadian model, the asynchronous strategy still gives a single attractor, but the terminal SCC is now composed of 442 states and 1737 transitions. Interestingly, the five states of the synchronous attractor are all present in the asynchronous attractor, but the simple cyclic trajectory has now been replaced by a complex graph with a much larger amount of details. To further analyze this attractor and validate our model, we consider six different versions of the model representing six known mutations (see Table 3). For some of them, circadian oscillations may be completely lost, which in our model translates to the attractor shrinking to a single fixed state. For others, oscillations endure but are somewhat degraded (eg. with a shorter period); in those cases our model conserves a complex attractor, but with less states than the original. These results seem to confirm that the model in Table 2 reproduces the essential core of the circadian clock dynamics.

Table 3. Effects of some mutations on the dynamics of the Boolean model.

\begin{tabular}{|c|c|c|}
\hline Mutation & Biological phenotype & Effect on the attractor \\
\hline$\overline{\mathrm{Bmal}}=0$ & $\begin{array}{l}\text { Arrhythmic (complete loss of cir- } \\
\text { cadian oscillations) }[9,18,19]\end{array}$ & Single state attractor 0100100000 \\
\hline $\mathrm{PER}=0$ & $\begin{array}{l}\text { Abnormal circadian oscillations } \\
{[9,18,19]}\end{array}$ & Attractor of 114 states \\
\hline $\mathrm{CRY}=0$ & $\begin{array}{l}\text { Abnormal circadian oscillations } \\
{[9,18,19]}\end{array}$ & Attractor of 106 states \\
\hline $\mathrm{REV}=0$ & Shorter cycle period $[15,18]$ & Attractor of 80 states \\
\hline $\mathrm{ROR}=0$ & Arrhythmic $[9,18]$ & Single state attractor 00000000000 \\
\hline $\mathrm{REV}=1$ & Arrhythmic $[10]$ & Single state attractor 00100000000 \\
\hline
\end{tabular}

Mutant analysis constitutes an interesting way to validate a discrete dynamical model. Indeed, the comparison between wild type and mutant phenotypes usually provides qualitative differences, such as the disappearance of oscillations for instance, that can be well captured by a Boolean model. Nevertheless, when dealing with such a complex attractor (hundreds of states, thousands of transitions), a more direct comparison of the attractor with biological data is rapidly limited, hindering model validation. For example, in Table 3 the degradation of circadian oscillations is paralleled with the number of states in the attractor, which is questionable. In the following we use the methodology proposed in [5], constructing a reduced version of the attractor based on biological knowledge. This summary graph leads to refine the analysis of the attractor, providing further validation of the model. 


\section{Comparing the attractor with circadian oscillations}

\subsection{Dividing the circadian cycle into qualitative phases}

In order to further analyze the model's attractor, a necessary first step is to classify its states into groups that will correspond to different stages of the circadian clock. This is essentially a modeling step, therefore there is not a unique way to make this classification, as it is based on a compromise between available biological data on the one hand, and the different variables and interactions included in the model on the other. In the following, we give a brief description of the main regulatory events during the circadian cycle, together with the modeling choices we made to deduce the corresponding partition of the attractor's states. For a comprehensive biological review we mainly referred to [20]. Note that this article is based on data at the transcriptional level; therefore, we sometimes used other sources to complete our classification (see $[2,16]$ and references therein).

As already mentioned, a hallmark of circadian rhythm progression is the phase opposition between the CLOCK:BMAL1 complex on the one hand and the PER:CRY complex on the other. This opposition divides the cycle into two major steps, approximately correlated with the day/night separation (see Figure 2). More precisely, [20] introduces two biological phases, respectively called Activation and Repression. The first one takes place during the day and corresponds to the activation of Bmal, while PER:CRY is absent. The second one sees the repression of Bmal and takes place during the night. Projecting this on the variables of our model, this leads to consider two groups of states: one where $B m a l=1, P C=0$ and one where Bmal $=0, P C=1$.

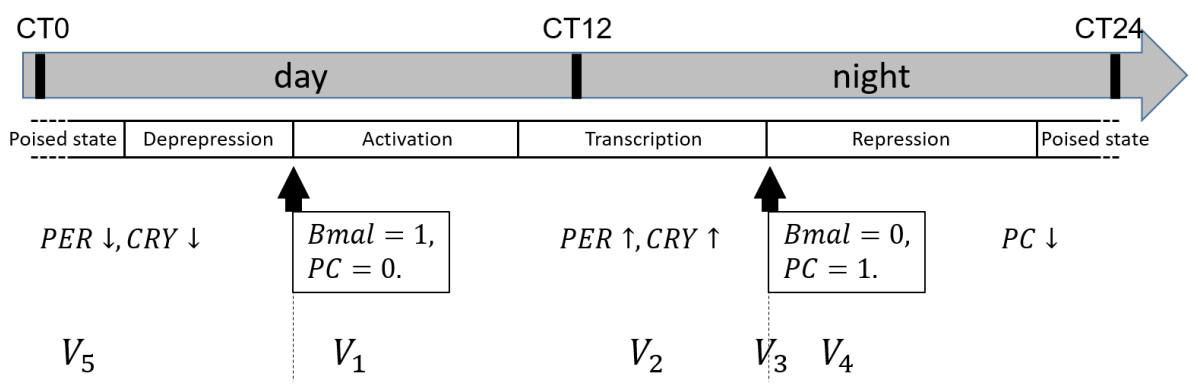

Fig. 2. Description of the main qualitative stages of the circadian clock. CT stands for Circadian Time and is an standard marker of time arbitrarily starting (CT0) at the beginning of activity for a diurnal organism. Top: temporal succession of biological phases as described in [20]; middle: projection of the main regulatory events on the model's variables; bottom: corresponding qualitative phases $V_{i}$ defined in (1).

These two main phases are separated by intermediate phases called Transcription and Poised state-Derepression in [20]. In the Transcription phase, Per 
and Cry genes are transcribed, followed by their complexation and the translocation of the complex into the nucleus. With the lack of precise timing of these events, we decided to subdivide this into a first step where PER or CRY are present but not at the same time, followed by a second step where they are both present. The latter corresponds to the pre-formation step of the PER:CRY complex, i.e. PER and CRY have sufficiently accumulated and the complex is about to form in the cytosol [19]. Finally, after the repression phase, the PER:CRY complex disappears due to auto-repression of PER and CRY [8]. Again, without knowing the precise timing of the disappearance of PER, CRY and the complex, we simply consider intermediary states where $\mathrm{Bmal}=P C=P E R=C R Y=0$, just before the activation of Bmal and the subsequent beginning of a new cycle.

This description leads to the definition of five groups of states in the attractor, or qualitative phases, denoted by $\left(V_{i}\right)_{1 \leq i \leq 5}$ and defined as follows.

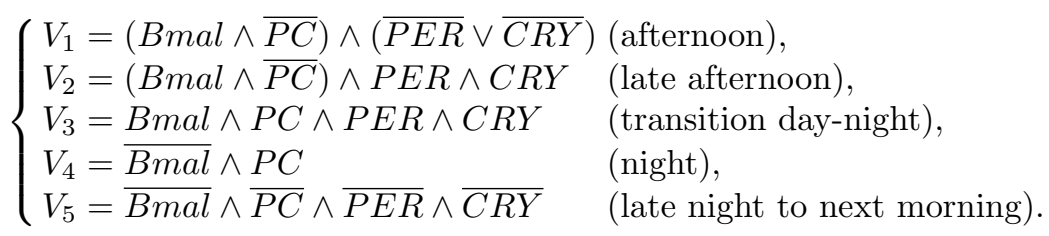

These phases are defined by taking into account the main variables of our Boolean model. They are based on qualitative and not temporal considerations; however, thanks to the description in [20] we were able to approximately place them along the circadian time scale (see Figure 2).

Remark 1. For the sake of convenience, we use the same symbol $V_{i}$ to designate both the subset of states in the attractor and the Boolean formula describing those states. For instance, $V_{4}=\overline{B m a l} \wedge P C$ denotes the set of states in the attractor such that $B m a l=0$ and $P C=1$.

\subsection{Construction of the summary graph}

Let $A=(V, E)$ denote the attractor, which is a directed graph over $|V|=$ 442 states. The sets $V_{i}$ defined by (1) are, by definition, subsets of $V$ that are mutually exclusive. To complete them into a partition of $V$, introduce the set $U=V \backslash\left(\bigcup_{i=1}^{5} V_{i}\right)$, containing "unclassified" states. The first thing to note with this partition is that every $V_{i}$ is actually not empty, confirming the attractor accurately captures all important phases of the circadian clock. To be more precise, $\left|V_{1}\right|=77,\left|V_{2}\right|=31,\left|V_{3}\right|=31,\left|V_{4}\right|=136$ and $\left|V_{5}\right|=30$ and there are $|U|=137$ unclassified states. We now briefly recall the definition of a summary graph (interested reader may refer to [5] for more details).

Definition 1. The summary graph of the graph $A=(V, E)$ on a partition $\mathcal{P}=\left\{V_{1}, V_{2}, \ldots, V_{k}\right\}$ of $V$ is the directed graph $\mathcal{G}=(\mathcal{P}, \mathcal{E})$, whose vertices are the $V_{i}, i=1, \ldots, k$ and where there is an edge from $V_{i}$ to $V_{j}$ iff $i \neq j$ and there exist $x \in V_{i}$ and $y \in V_{j}$ such that $(x, y) \in E$. 
From a graph theoretical point of view, $\mathcal{G}$ is simply the quotient graph of $A$ on the partition $\mathcal{P}$. For the sake of simplicity, we use the same symbol $V_{i}$ to design the set of states $V_{i}$ and the vertex $V_{i}$ of the summary graph. This enables an easy description of sets of trajectories in the attractor $A$ : actually, each edge of the summary graph is unequivocally associated with a subset of asynchronous transitions in $A$. The summary graph of the attractor is depicted in Figure 3 (top left).
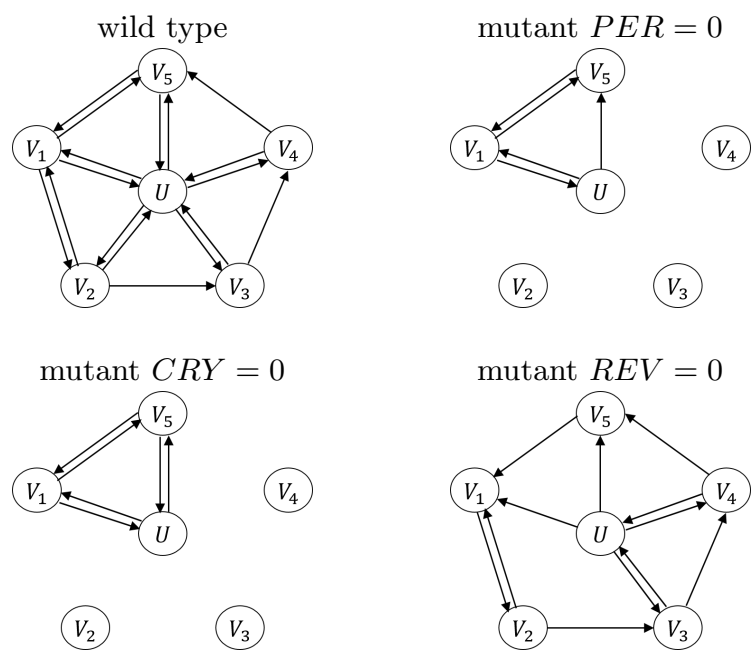

Fig. 3. Summary graph of the attractor on the partition $\left\{U, V_{i}, i=1 \ldots 5\right\}$ defined in (1), in wild type condition as well as for mutants $P E R=0, C R Y=0$ and $R E V=0$.

The summary graph in Figure 3(top left) shows that the expected succession of phases $V_{1} \rightarrow V_{2} \rightarrow V_{3} \rightarrow V_{4} \rightarrow V_{5} \rightarrow V_{1}$ is actually present, confirming the existence of accurate circadian oscillations in the attractor, with respect to regulators Bmal, PER, CRY and PER:CRY. Moreover, three one-directional transitions $V_{2} \rightarrow V_{3}, V_{3} \rightarrow V_{4}$, and $V_{4} \rightarrow V_{5}$ even indicate irreversible progression through the cycle. The edge $V_{2} \rightarrow V_{3}$ corresponds to the formation and translocation of the PC complex, following the accumulation of PER and CRY. This irreversibility is in adequacy with [19]: as the main role of $P C$ is to inhibit Bmal, it will switch off only after $B m a l=0$. Similarly, the edge from $V_{3}$ to $V_{4}$ corresponds to transitions where $\mathrm{Bmal}$ is repressed, which is known to be irreversible. As for the edge $V_{4} \rightarrow V_{5}$, it is associated to a set of transitions where $P C$ switches off and can be viewed as a consequence of PER and CRY auto-repression [8]. From a graph point of view, the presence of these three one-directional edges imposes a general orientation for the cycle, consistent with what is known on the opposition between the Activation and Repression phases of $[20]$. 
In addition to the summary graph in wild type condition, Figure 3 also shows the summary graphs for the three mutants that exhibited oscillatory behaviors in Table 3: $P E R=0, C R Y=0$ and $R E V=0$. Interestingly, the mutation of $R E V$ has a limited impact on the succession of the phases, showing this perturbation does not impair the cycle progression, as it was observed in $[15$, 18]. For $P E R$ and $C R Y$ mutants, the perturbation is stronger and the cycle is impaired. However, we still observe a $\mathrm{Bmal}$ oscillation between $V_{1}$ and $V_{5}$ (notably through $U$ ), confirming circadian oscillations may persist, although in a degraded form $[9,18,19]$.

Whether in wild type or in mutant conditions, the summary graph reveals to be a powerful tool to analyze asynchronous attractors of complex oscillatory biological systems, such as the circadian clock. In the next part, we propose an extension of this analysis, where we show how to use this tool to help redesign the network, and assess the general robustness of the model.

\section{Advanced analysis of the attractor}

The summary graphs in Figure 3 allow a direct comparison of the model's attractor with biological knowledge on the circadian cycle, both in wild type and mutant conditions. In particular, we were able to track the expected succession of qualitative phases within the attractor, thus validating that our model captures the essential core of the regulatory network. Nevertheless, the summary graphs also point to spurious transitions to (and from) the set of unclassified states $U$, responsible for "short-circuits" between phases. Such spurious trajectories may reveal abnormal behaviors, with unwanted transitions in the model, or they may catch important links ensuring the overall robustness of the cycle. In both cases, the summary graph provides an ideal tool to detect those spurious transitions and analyze them in a biological context.

\subsection{Adjustment of the attractor and refinement of the model}

We start with a more in-depth examination of the set $U$. In part 3 it is defined as $U:=V \backslash\left(\bigcup_{i} V_{i}\right) \subseteq\{0,1\}^{11}$ and is a subset of 137 states in the attractor that we were not able to classify according to the general description of the circadian cycle. By assigning the value 1 to the states in $U$ and the value 0 to the states in $V \backslash U$, we obtain a partial Boolean function (PBF) that we can identify using Boolean inference techniques (see $[21,22]$ ). The inference consists in identifying minimal supports ${ }^{3}$, i.e. minimal subsets of variables that are sufficient to reproduce the PBF. This function provides an easy way to characterize the

\footnotetext{
${ }^{3}$ In [21], this step is performed by the algorithm REVEAL [11], in [22] it is performed by the algorithm presented in [12]. Overall, all the inferences performed in the present paper are fast, due to the small dimension (11) of the network. A general discussion about the complexity of the inference problem can be found for instance in [22].
} 
unclassified states, showing they can be decomposed into two components:

$$
U=\underbrace{\overline{B m a l} \wedge \overline{P C} \wedge(P E R \vee C R Y)}_{U_{1}} \vee \underbrace{B m a l \wedge P C \wedge \overline{P E R}}_{U_{2}} .
$$

While the inference of a PBF does not lead to a unique solution in general, here (2) is actually unique, as the set $\{\mathrm{Bmal}, \mathrm{PC}, \mathrm{PER}, \mathrm{CRY}\}$ is the only minimal support of the PBF $U$ (it will be the case for all PBF identified in this article). As before, we conveniently use the same symbol $U$ to designate the set of unclassified states (within the attractor) and the Boolean function that characterizes them (see Remark 1 above).

The Boolean formula of $U$ is decomposed as in (2) to highlight two separate subsets of unclassified states: the first one $U_{1}$ is composed of states where variables $\mathrm{Bmal}$ and $P C$ are both off whereas the second one $U_{2}$ is composed of states where variables $B m a l$ and $P C$ are both on. Specifically, in this second subset the PER:CRY complex is forming (since it has not repressed Bmal yet) while PER is switched off. Clearly, such states should not exist in the attractor since PER is essential for complex formation. In order to observe the interplay between $U_{1}, U_{2}$ with the different phases $V_{i}$, we reconstruct the summary graph of the attractor, using Definition 1 on the new partition $\left\{V_{1} \ldots, V_{5}, U_{1}, U_{2}\right\}$ (Figure 4, left).

By looking at this graph, one can see that the set $U_{2}$ is connected to the rest of the attractor mainly through phase $V_{3}$. More precisely, only six transitions in the attractor are responsible for the entry into $U_{2}$, all coming from $V_{3}$. Since an asynchronous strategy is used, all these transitions can be traced back to situations where PER has disappeared before the complex PER:CRY has completed the repression of Bmal, leading to a contradiction. Therefore, we decide to suppress these transitions in the attractor, leading to the disconnection of the set $U_{2}$ (Figure 4, center).
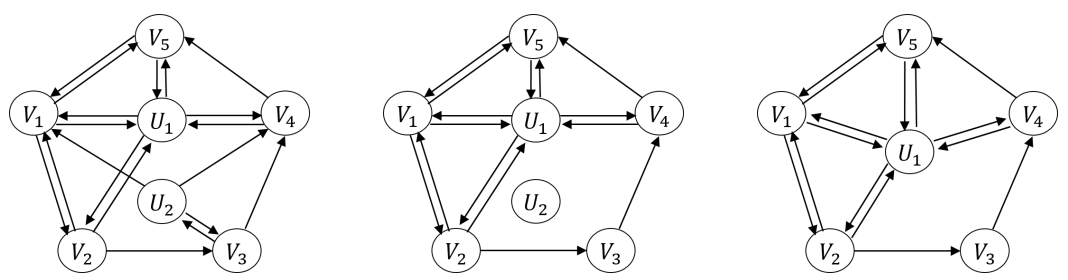

Fig. 4. Refined summary graphs. Left: initial attractor's summary graph with $U$ decomposed in $\left(U_{1}, U_{2}\right)$; center: summary graph once the six transitions $V_{3} \rightarrow U_{2}$ have been removed; right: summary graph of the new amended model's attractor.

After the removal of the six transitions in the graph of the attractor, we obtain a new amended model by applying a few steps. First, we recompute the strongly connected component decomposition of the truncated attractor. We find a unique terminal SCC of 393 states, in which the states in $U_{2}$ have disappeared. 
From this SCC we reconstruct the partial truth table of the network on these 393 states, and then use an inference technique to identify the logical rules corresponding to this partial truth table. The last two steps (construction of partial truth table, followed by inference) are described in more details in [5] and, in a different context, in [21]. Finally, the only affected rule of the network is the rule of variable $P E R$, with the addition of a new (unique) clause:

$$
P E R^{\prime}=(B m a l \wedge \overline{C R Y 2}) \vee(D B P \wedge \overline{E 4}) \vee(\mathbf{B m a l} \wedge \mathbf{P C}) .
$$

To verify that this modification did not alter other parts of the dynamics, we made the same analyses as in Parts 2 and 3, confirming that the slight modification in (3) was sufficient to get rid of unwanted states $U_{2}$ while conserving good dynamical properties, in wild type and mutant conditions. The summary graph of this new amended model is depicted in Figure 4, on the right.

The modified rule (3) adds a single new interaction in the network, which is a positive effect of $P C$ onto PER (the positive effect of $B$ mal to $P E R$ was already present in the original network). Interestingly, this effect was present in the ODE system of [2], as an unbinding term of the PER:CRY complex. As already said, such mass action law kinetic terms are often made implicit by default in the Boolean framework. Here, our method points to the importance of this particular one to avoid unwanted transitions within the attractor. From a modeling point of view, the summary graph thus provides a valuable help in the design of Boolean models of complex, oscillating biological systems.

\subsection{A tool to assess the general robustness of the attractor}

The remaining set of unclassified states $U_{1}$ is composed of states where $B$ mal and $P C$ are off while PER or $C R Y$ can be on. Contrary to $U_{2}$, this set is highly connected to almost every phases of the attractor (see Figure 4). In total, the four edges entering in $U_{1}$ amount to 117 transitions. As before, we tried to remove those transitions in order to disconnect $U_{1}$ from the rest of the attractor, thus obtaining a final summary graph perfectly matching the order of the phases. However, although showing no more short-cut between phases, this version loses some essential dynamical property. Indeed, the PER and CRY mutants no longer exhibit oscillatory behaviors, suggesting that at least a subset of these transitions are needed for a proper behavior of the model.

Nevertheless, even though those transitions cannot be removed all together, we can still use the summary graph to investigate the model further and analyze each edge entering $U_{1}$ separately, in the context of the circadian cycle.

1. The edge $V_{4} \rightarrow U_{1}$ is associated with 63 transitions in which the variable $P C$ switches off while $P E R$ and $C R Y$ are not both deactivated. From a biological point of view, it means that the PER:CRY complex disappears before PER and CRY have disappeared, indicating a short half-life of the complex. These transitions are to be compared with the $V_{4} \rightarrow V_{5}$ transitions where $P C$ switches off after $P E R$ and $C R Y$, suggesting a longer half-life. 
In our model the two types of transitions coexist since the relative times of disappearance of the three components, PER, CRY and PER:CRY are not taken explicitly into account. Interestingly, note that the removal of the 63 transitions does not affect the global dynamical properties of the attractor, as the mutants are not affected.

2. The edge $V_{5} \rightarrow U_{1}$ corresponds to 9 transitions in the attractor, in which the variable $P E R$ switches on while $B m a l$ is not yet active. Since PER transcription is activated through the CLOCK:BMAL1 complex, these transitions seem rather unrealistic. To investigate further, we removed the transitions and applied the same technique as before, to obtain the modified $P E R$ rule:

$$
\begin{aligned}
P E R^{\prime}=(B m a l \wedge \overline{C R Y 2}) & \vee(B m a l \wedge P C) \\
& \vee[(D B P \wedge \overline{E 4}) \wedge(\mathbf{P E R} \vee \mathbf{C R Y} \vee P C)] .
\end{aligned}
$$

Dynamically, the removal does not affect the main properties of the attractor (the summary graphs of wild type and mutant conditions are similar) however, the inferred logical rule exhibits new and undocumented interactions, namely an auto-activation of PER and a positive effect of CRY on PER. This points to a specific part of the model that will need a closer look in the future.

3. Finally, the edges $V_{1} \rightarrow U_{1}$ and $V_{2} \rightarrow U_{1}$ correspond to a total of 45 transitions in which the variable $B m a l$ switches to 0 in the absence of $P C$. With respect to circadian clock events, these transitions describe an early deactivation of Bmal, leading the cycle to bypass the important steps of PER:CRY formation and translocation (phase $V_{3}$ ). However, it is the removal of these transitions that directly alter the behaviors of the mutants, suggesting they are necessary to ensure the robustness of the attractor. Their removal leads to a new rule for Bmal:

$$
B m a l^{\prime}=(R O R \wedge \overline{R E V} \wedge \overline{P C}) \vee[(\mathbf{B m a l} \wedge(\mathbf{P E R} \vee \mathbf{C R Y}) \wedge \overline{\mathbf{P C}}],
$$

that highlights, besides an auto-activation term, a direct positive effect of $P E R$ and $C R Y$ on Bmal. Those new interactions generate two positive feedback loops involving Bmal, PER and $C R Y$ that directly interfere with the negative feedback loop between $\mathrm{Bmal}$ and $P C$ :

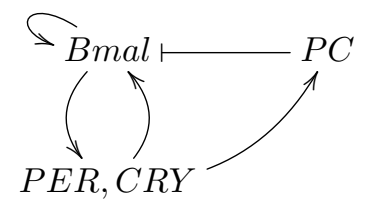

This alteration of the negative loop is directly responsible for the loss of robustness of the model.

The objective of this section was to analyse the "short-cut" transitions in the circadian attractor (see Fig. 4 left). As shown by the three examples above, the summary graph combined with Boolean inference indicates that some of these 
unwarranted transitions should not be removed and, in fact, appear to play a significant role during the progression of the cycle, namely in the system's response to gene knock-outs.

\section{Conclusions and perspectives}

In this article we used the summary graph, a novel tool introduced in [5], to analyze a Boolean model of the mammalian circadian cycle regulation. This tool is well adapted to study biological oscillations, as it provides a rational way to compare a complex Boolean attractor (with hundreds of states) with an oscillating phenomenon. Combined with Boolean inference techniques, it becomes particularly useful in model design as it allows to relate a local dynamical property of the attractor, such as the transition from one phase to the next, to the part of the network's topology directly responsible for it.

After verifying the model's attractor correctly captures the correct succession of phases, the summary graph was further analyzed to provide more insight on the model. In a first step, our analysis indicates that the attractor contains many "short-cuts", that is transitions between states (essentially towards sets $U_{1}$ and $U_{2}$ ) which may lead to distorted cycles with very short days or very short nights. In a second step, the analysis shows that some of these short-cuts (those passing through set $U_{2}$ ) can be removed by a refinement of the logical rules. Namely, the rule of PER should be modified to include the effect of PC dissociation which, for simplicity, was not taken into account in the initial Boolean model (compare Table 2 and equation (3)). Finally, in a third step, our analysis suggests that some short-cuts, specifically $V_{1} \rightarrow U_{1}$ and $V_{2} \rightarrow U_{1}$, are necessary for a correct performance. These transitions are characterized by an early BMAL1 deactivation, and they are responsible also for generating the short cycle mutants observed in the PER and CRY knock-outs. The transitions through $U_{1}$ may thus be necessary to generate circadian cycle robustness in response to perturbations in gene expression.

More generally, the summary graph provides an efficient way to tackle complex qualitative attractors, by testing the effect of specific perturbations on the dynamics. In future works we plan to further investigate the role of the states $U_{1}$ in maintaining circadian oscillations, by studying the links between state transitions and the topology of the circadian network.

\section{Acknowledgements}

The authors would like to thank Franck Delaunay for many useful discussions on the circadian clock events, as well as for pointers to relevant references.

\section{References}

1. Akman, O., Watterson, S., Parton, A., Binns, N., Millar, A., Ghazal, P.: Digital clocks: simple boolean models can quantitatively describe circadian systems. J. R. Soc. Interface 9, 2365-2382 (2012) 
2. Almeida, S., Chaves, M., Delaunay, F.: Transcription-based circadian mechanism controls the duration of molecular clock states in response to signaling inputs. Journal of theoretical biology 484, 110015 (2020)

3. Chaves, M., Tournier, L., Gouzé, J.L.: Comparing Boolean and piecewise affine differential models for genetic networks. Acta biotheoretica 58(2-3), 217-232 (2010)

4. Comet, J.P., Bernot, G., Das, A., Diener, F., Massot, C., Cessieux, A.: Simplified models for the mammalian circadian clock. Procedia Computer Science 11, 127$138(2012)$

5. Diop, O., Tournier, L., Fromion, V.: Summarizing complex asynchronous Boolean attractors, application to the analysis of a mammalian cell cycle model. In: 18th European Control Conference (ECC), Naples, Italy. pp. 1677-1682 (2019)

6. Fauré, A., Naldi, A., Chaouiya, C., Thieffry, D.: Dynamical analysis of a generic boolean model for the control of the mammalian cell cycle. Bioinformatics 22(14), e124-e131 (2006)

7. Forger, D.B.: Biological clocks, rhythms, and oscillations: the theory of biological timekeeping. MIT Press (2017)

8. Gallego, M., Virshup, D.M.: Post-translational modifications regulate the ticking of the circadian clock. Nature reviews Molecular cell biology 8(2), 139-148 (2007)

9. Ko, C.H., Takahashi, J.S.: Molecular components of the mammalian circadian clock. Human molecular genetics 15(suppl_2), R271-R277 (2006)

10. Kornmann, B., Schaad, O., Bujard, H., Takahashi, J.S., Schibler, U.: System-driven and oscillator-dependent circadian transcription in mice with a conditionally active liver clock. PLoS biology 5(2) (2007)

11. Liang, S., Fuhrman, S., Somogyi, R.: Reveal, a general reverse engineering algorithm for inference of genetic network architectures. In: Pacific Symposium on Biocomputing. vol. 3, pp. 18-29 (1998)

12. Murakami, K., Uno, T.: Efficient algorithms for dualizing large-scale hypergraphs. Discrete Applied Mathematics 170, 83-94 (2014)

13. Ndiaye, I., Chaves, M., Gouzé, J.L.: Oscillations induced by different timescales in signal transduction modules regulated by slowly evolving protein-protein interactions. IET systems biology 4(4), 263-276 (2010)

14. Poignard, C., Chaves, M., Gouzé, J.L.: A stability result for periodic solutions of nonmonotonic smooth negative feedback systems. SIAM Journal on Applied Dynamical Systems 17(2), 1091-1116 (2018)

15. Preitner, N., Damiola, F., Zakany, J., Duboule, D., Albrecht, U., Schibler, U., et al.: The orphan nuclear receptor rev-erb $\alpha$ controls circadian transcription within the positive limb of the mammalian circadian oscillator. Cell 110(2), 251-260 (2002)

16. Relógio, A., Westermark, P.O., Wallach, T., Schellenberg, K., Kramer, A., Herzel, H.: Tuning the mammalian circadian clock: robust synergy of two loops. PLoS computational biology $\mathbf{7}(12)$ (2011)

17. Remy, E., Mossé, B., Thieffry, D.: Boolean dynamics of compound regulatory circuits. In: Dynamics of Mathematical Models in Biology, pp. 43-53. Springer (2016)

18. Ripperger, J.A., Jud, C., Albrecht, U.: The daily rhythm of mice. FEBS letters 585(10), 1384-1392 (2011)

19. Rosensweig, C., Green, C.B.: Periodicity, repression, and the molecular architecture of the mammalian circadian clock. European Journal of Neuroscience 51(1), 139165 (2018)

20. Takahashi, J.S.: Transcriptional architecture of the mammalian circadian clock. Nature Reviews Genetics 18(3), 164 (2017) 
21. Tournier, L., Chaves, M.: Uncovering operational interactions in genetic networks using asynchronous Boolean dynamics. Journal of theoretical biology 260(2), 196209 (2009)

22. Tournier, L., Goelzer, A., Fromion, V.: Optimal resource allocation enables mathematical exploration of microbial metabolic configurations. Journal of mathematical biology 75(6-7), 1349-1380 (2017)

23. Traynard, P., Feillet, C., Soliman, S., Delaunay, F., Fages, F.: Model-based investigation of the circadian clock and cell cycle coupling in mouse embryonic fibroblasts: Prediction of reverb- $\alpha$ up-regulation during mitosis. BioSystems 149, 59-69 (2016)

24. Van Ham, P.: How to deal with variables with more than two levels. In: Kinetic Logic a Boolean Approach to the Analysis of Complex Regulatory Systems, pp. 326-343. Springer (1979) 